\title{
Valutazione della comprensione dell'ascolto e del testo scritto nella scuola elementare
}

\author{
Lucia Allegra \\ Scuola Elementare Dante Alighieri, Isola, Slovenia \\ allegralucia14@gmail.com \\ Sergio Crasnich \\ Istituto dell'Educazione della Repubblica di Slovenia \\ sergio.crasnich@zrss.si \\ Nives Zudič Antonič \\ Università del Litorale, Slovenia \\ nives.zudic.antonic@fhs.upr.si
}

Il contributo presenta una ricerca quantitativa avente come obiettivo il controllo degli effetti di potenziamento, esercitabili sulla comprensione della lettura e dell'ascolto da un intervento didattico. La scelta del tema è motivata dall'importanza di tali abilità ricettive nei processi di insegnamento e apprendimento. Alla ricerca ha partecipato un gruppo di alunni frequentanti una scuola elementare con lingua d'insegnamento italiana sul territorio nazionalmente misto dell'Istria slovena. Il lavoro supporta l'opportunità di adottare precocemente percorsi di intervento di carattere metacognitivo ricorrendo all'uso di prove oggettive e standardizzate, utili a fornire informazioni precise sui processi di comprensione degli alunni e sulle difficoltà cui essi possono andare incontro.

Parole chiave: educazione linguistica, abilità ricettive, comprensione, prove standardizzate, analisi

(cc)BY-SA https://doi.org/10.26493/978-961-293-045-5.93-109

\section{Introduzione}

Comprendere ciò che si ascolta o ciò che si legge è un aspetto centrale della vita quotidiana e, per converso, difficoltà o lacune nella comprensione hanno effetti negativi sulla nostra capacità di affrontare adeguatamente le più diverse situazioni della vita scolastica, lavorativa e personale. Nell'ambito dell'istruzione la comprensione rappresenta un fattore cruciale, in quanto studiare significa prima di tutto comprendere un'informazione per poi poterla ricordare; di conseguenza, la disponibilità di adeguate abilità di comprensione è una delle principali condizioni del successo scolastico.

I processi che intervengono durante la comprensione sono numerosi e variamente interagenti tra loro. Ogni individuo è condizionato dal pro- 
prio bagaglio linguistico ed esperienziale, nonché dal proprio stato affettivo. Alcune componenti di tali processi possono essere oggetto di interventi didattici basati sul riferimento al costrutto di metacognizione, miranti a promuovere l'attivazione di strategie di pianificazione e controllo consapevole.

Il presente contributo si concentra sulla comprensione dell'ascolto e del testo scritto in alunni di terza, quarta e quinta elementare, frequentanti una scuola con lingua d'insegnamento italiana sul territorio nazionalmente misto dell'Istria slovena. ${ }^{1}$ Attraverso il ricorso a un intervento didattico strutturato e la somministrazione di prove standardizzate, ci si è posti l'obiettivo di potenziare le strategie utilizzate dagli alunni nel processo di comprensione.

Nel territorio bilingue dell'Istria slovena, dove la ricerca è stata svolta, l'italiano è lingua ufficiale accanto allo sloveno ed è lingua d'ambiente tutelata dalle leggi dello stato. Sul territorio si sono aggiunte alla cultura slovena anche diverse culture (croata, serba, bosniaca, albanese, macedone) legate a movimenti di migrazione interna (Zudič Antonič 2007, 26) e, più di recente, internazionale (Crasnich 2019, 265).

In conseguenza di ciò, negli ultimi anni le scuole con lingua d'insegnamento italiana stanno registrando sia un aumento del numero di alunni, sia una crescente presenza al loro interno di alunni non italofoni (Crasnich 2019, 264). Benché non tutti gli alunni condividano l'italiano come lingua materna, l'adozione di metodi implicanti la stimolazione di abilità metacognitive legate alla L1 si giustifica in virtù della elevata esposizione degli alunni alla lingua italiana, nonché della loro precoce immersione nell'ambiente linguistico italofono per il tramite della scuola dell'infanzia (Crasnich 2019, 264).

All'ingresso nella scuola primaria, quindi, si può assumere che gli alunni abbiano già sviluppato competenze linguistiche, comunicative e pragmatiche abbastanza elevate che, in termini neuropsicologici, costituiscono i moduli neuro-funzionali maggiormente sviluppati. Se l'acquisizione delle competenze pragmatiche e linguistiche risulta già in qualche modo avviata (Daloiso 2009, 36), la piena maturazione della competenza metalinguistica necessita invece di maggiore supporto.

\footnotetext{
${ }^{1}$ Nella Repubblica di Slovenia, la scolarizzazione elementare inizia mediamente a sei anni di età. La scuola elementare è articolata in tre cicli triennali, per una durata complessiva di nove anni. L'età dei partecipanti alla ricerca era compresa tra gli otto e i dieci anni.
} 


\section{La comprensione del testo, dall'ascolto alle abilità cognitive}

Numerosi studi hanno evidenziato l'importanza della comprensione dell'ascolto per la comprensione del testo scritto; nelle fasi iniziali dell'apprendimento, il livello di comprensione è maggiormente spiegato dall'efficienza nella lettura ad alta voce, che costituisce per il lettore una forma di ascolto di sé. La correlazione tra decodifica e comprensione diminuisce al crescere dell'età, in quanto l'abilità di lettura si automatizza (Carretti et al. 2013, 8).

Per far acquisire ai discenti una adeguata padronanza dei meccanismi legati alla comprensione è necessario promuovere lo sviluppo dei processi cognitivi implicati sia nell'ascolto sia nella lettura. Il modello cognitivista di elaborazione delle informazioni ha sottolineato l'importanza, per il processo di comprensione, di integrare efficacemente tra loro due tipi di processi, definiti metaforicamente bottom up (dal basso verso l'alto) e top down (dall'alto verso il basso): in sostanza, il soggetto parte dagli stimoli letti o ascoltati, integrando i processi di decodifica delle informazioni provenienti dai testi con processi di interpretazione attiva di queste ultime, legati all'impiego di strutture di conoscenza già depositate nella memoria sotto forma di rappresentazioni dette schemi. Un approccio didattico basato sulla promozione e sul ricorso al tipo di operazioni di integrazione appena descritte è costituito dalla "grammatica dell'anticipazione» (expectancy grammar), termine coniato da Oller (Balboni 2007, 19) e che Balboni (2012, 171) considera una componente fondamentale del processo di comprensione. La grammatica dell'anticipazione è un processo mirato e finalizzato, che non si attiva in modo generalizzato; essa, infatti, è orientata dagli scopi dell'individuo che si appresta ad ascoltare un discorso, o a leggere un testo. In base allo scopo per il quale si vuole comprendere un testo (piacere, informazione, studio) si utilizzano strategie diverse, che permettono ai meccanismi coinvolti nell'expectancy grammar il compito di formulare ipotesi utilizzando un numero inferiore di variabili. Un obiettivo specifico dell'educazione linguistica è quindi di insegnare quale tipo di strategia sia più efficace, e come una strategia funzioni (Balboni 1994, 37).

Il lettore o l'ascoltatore possono avvicinarsi alla lettura o all'ascolto di un testo con obiettivi precisi, che possono variare a seconda del contesto: di conseguenza, diverse saranno anche le modalità di comprensione - globale, analitica, selettiva, approfondita (Daloiso 2011, 58). Ciò assume particolare e decisiva importanza in ambito scolastico, dove la lettura è prevalentemente finalizzata allo studio: studiare, infatti, significa innanzitutto comprendere quello che si apprende per poterlo ricordare (Carretti et al. 
2013) sviluppando consapevolmente adeguate strategie di controllo. Comportando l'attivazione di strategie, la lettura va intesa come un processo cognitivo e metacognitivo, che coinvolge funzioni psichiche quali l'attenzione, la classificazione, la categorizzazione, la memoria (Ferraboschi e Meini 1993).

Come osservato da Albanese, Doudin e Martin $(2003,49)$ il termine metacognizione, inteso come conoscenza dei propri processi cognitivi e dei loro risultati, nonché di ciò che a essi è collegato, ha origine negli anni Settanta del secolo scorso. Tra gli autori italiani, Cornoldi e Caponi (1991, 10-11) definiscono la metacognizione come l'insieme delle attività psichiche che presiedono al funzionamento cognitivo, acquisito attraverso le esperienze compiute durante lo svolgimento dei processi cognitivi e le attività di riorganizzazione cui essi danno luogo. Cornoldi e Caponi (1991) distinguono tra conoscenza metacognitiva (insieme delle conoscenze possedute dall'individuo sul funzionamento della mente) e processi metacognitivi di controllo (insieme di attività che presiedono ai processi cognitivi durante la loro esecuzione). Una simile distinzione, che chiama in causa la consapevolezza e il controllo esecutivo, è proposta anche da Boscolo (2006): secondo tale autore, quest'ultimo permette la scelta della strategia più appropriata per eseguire una particolare fase di un compito.

L'indagine metacognitiva si è focalizzata maggiormente sugli ambiti della lettura e della memoria. Molte ricerche hanno dimostrato che la metacognizione occupa un ruolo fondamentale nella cognizione del testo e nel ricordo di ciò che è stato letto. Inoltre, numerosi autori concordano nel ritenere che le strategie metacognitive siano insegnabili: in base a questo presupposto, il principale obiettivo dell'insegnamento diviene quello di far acquisire abilità specifiche, utilizzate dai buoni lettori ma non padroneggiate da tutti gli allievi (Albanese, Doudin e Martin 2003, 187). Si ritiene, infatti, che un lettore consapevole dei propri processi cognitivi possa giungere a una comprensione adeguata grazie alla conoscenza di strategie efficaci, delle caratteristiche dei testi e dei propri punti di forza e di debolezza (Albanese, Doudin e Martin 2003, 178).

Il quadro teorico metacognitivo ha proposto a psicologi e a insegnanti un'alternativa ai modelli tradizionali di intervento didattico. Questo approccio, che attribuisce al lettore un ruolo centrale e attivo (Zanetti e Miazza 2004) implica che il processo educativo non debba riguardare solamente le abilità di base o già acquisite, o i prodotti dell'apprendimento, ma anche il modo in cui si realizzano la comprensione e l'utilizzo delle abilità stesse. Questo progredire al di là della cognizione mira soprattutto ad accresce- 
re nell'alunno la consapevolezza di ciò che sta facendo, del perché lo fa, di quando è utile farlo e in quali condizioni, per poi sviluppare la capacità di gestire i propri processi cognitivi guidandoli con valutazioni e indicazioni operative (Ianes 1996, 13).

\section{La ricerca}

\section{Premesse della ricerca e scelta del trattamento}

Il presente lavoro propone una ricerca quantitativa riguardante la comprensione dell'ascolto e del testo scritto nella scuola dell'obbligo. La scelta del tema è motivata dall'importanza di tali abilità ricettive nei processi di insegnamento e apprendimento. La maggior parte delle attività praticate a scuola, infatti, implica il ricorso al linguaggio orale; di conseguenza, buone competenze di comprensione dell'ascolto costituiscono una precondizione importante per un efficace funzionamento dei processi di integrazione e immagazzinamento delle informazioni. L'esigenza di verificare la comprensione del testo scritto, invece, è apparsa fondamentale poiché essa costituisce un aspetto essenziale dei processi di insegnamento e apprendimento. La comprensione del testo interessa tutte le discipline in cui si richiede l'uso di testi scritti, è in relazione con le abilità di studio ed è importantissima per il successo scolastico.

Le attività di potenziamento della comprensione dell'ascolto utilizzate nel presente lavoro riprendono le indicazioni proposte in CO-T T: comprensione orale (Carretti et al. 2013), che propone un percorso di miglioramento delle abilità associate alla comprensione del testo. Il rafforzamento si articola in 21 sessioni, dedicate alle conoscenze e al controllo metacognitivo, alla capacità della memoria di lavoro, all'integrazione delle informazioni (Carretti et al. 2013, 29).

Al termine del percorso è previsto lo svolgimento di prove di verifica della comprensione dell'ascolto di testi scritti, differenziate per ciascuna classe della scuola elementare, e di un testo audiovisivo, comune a tutte le classi. Ambedue i tipi di prove sono stati da noi utilizzati senza alcuna modifica, e somministrati seguendo le indicazioni date dagli autori.

Le prove di comprensione del testo scritto, somministrate nella presente ricerca, sono state scelte attingendo ai materiali elaborati dal gruppo di ricerca M T (Cornoldi, Colpo e Carretti, 2017) attivo presso il Dipartimento di Psicologia Generale dell'Università di Padova. Le prove comprendono testi per la valutazione delle abilità di lettura (correttezza e rapidità) e comprensione del testo. La valutazione da noi effettuata si è limitata alla comprensione del testo scritto; inoltre, poiché l'obiettivo era di indivi- 
duare la capacità di cogliere il contenuto delle informazioni presenti nel brano, agli alunni è stato consentito sia di leggere sileziosamente il testo al ritmo da loro scelto, sia di poterlo ricontrollare in qualsiasi momento della somministrazione. Per la presente ricerca sono state scelte le prove dell'indagine approfondita destinate alle classi di scuola primaria oggetto dell'intervento.

La standardizzazione del trattamento e delle prove utilizzate nel presente lavoro è stata effettuata su alunni di madrelingua italiana, frequentanti scuole della Repubblica italiana. Gli alunni che frequentano le scuole con lingua d'insegnamento italiano sulla costa slovena vivono in una realtà linguistica interculturale non paragonabile a quella italiana. Di conseguenza, le fasce di prestazione proposte dagli autori non sono state utilizzate a fini di confronto, ma solo come spunto utile alla formulazione di parte delle ipotesi di ricerca.

\section{Obiettivi della ricerca}

La ricerca si è posta i seguenti obiettivi:

1. favorire nei destinatari lo sviluppo di alcune strategie di comprensione dell'ascolto;

2. valutare le abilità ricettive in alunni di scuola primaria;

3. indagare il livello di competenze di comprensione della lettura;

4. promuovere, negli insegnanti di classe e di italiano della scuola elementare, l'utilizzo di prove standardizzate di lettura che, usate in diverse fasi dell'anno scolastico, potrebbero fornire informazioni sulle abilità di comprensione della lettura degli alunni, consentendo di adattare l'attività didattica alle effettive abilità e necessità di potenziamento degli alunni.

\section{Ipotesi di ricerca}

Per la ricerca sono state formulate le seguenti ipotesi:

1. le competenze dei partecipanti in tutte e tre le abilità testate potrebbero essere sufficientemente consolidate, ma i risultati nelle prove inferiori a quelli di alunni italiani e italofoni data la specificità del contesto scolastico oggetto della ricerca;

2. al crescere dell'età le abilità di comprensione dovrebbero migliorare, e quindi i risultati delle prove dovrebbero mostrare una tendenza all'aumento graduale dei punteggi dalla terza alla quinta; 
3. le competenze di ascolto e lettura potrebbero essere diverse tra un genere e l'altro, e in particolare il punteggio ottenuto dalle femmine potrebbe essere superiore a quello dei maschi.

\section{Partecipanti}

La ricerca ha coinvolto trentanove alunni di una scuola con lingua d'insegnamento italiana sul territorio nazionalmente misto dell'Istria slovena (tredici per ciascuna delle classi terza, quarta, quinta).

\section{Metodologia della ricerca}

La ricerca ha previsto sei incontri in ognuna delle tre classi, articolati in 3 fasi: (a) potenziamento delle abilità di ascolto - tre incontri della durata di 45 minuti in ogni classe, nel corso dei quali sono state svolte le attività di potenziamento del manuale CO-T T: comprensione orale (Carretti et al. 2013); (b) somministrazione delle prove di comprensione dell'ascolto proposte dalla batteria CO-T T, nel corso di due incontri della durata di 45 minuti per ciascuna classe; (c) somministrazione delle prove di comprensione del testo scritto proposte nel manuale Prove M T: kit scuola primaria (Cornoldi, Colpo e Carretti 2017).

\section{Risultati}

Di seguito sono presentati i risultati della ricerca. Va osservato che, a causa di assenze imprevedibili, il numero di alunni partecipanti alle diverse fasi della ricerca ha talora subito lievi variazioni.

La tabella 1 presenta i punteggi ottenuti nelle prove espressi in percentuale. Dai risultati ottenuti possiamo vedere che i punteggi minori sono stati registrati in terza e quarta nella prova audio-video (comune a tutti i gruppi), mentre la prestazione della quinta è sostanzialmente la medesima in tutte e tre le prove, e inferiore a quella delle altre due classi nelle prove scritta e di ascolto. I confronti tra le medie documentano il miglioramento dell'abilità di ascolto, attestato dall'incremento di prestazione al crescere

Tabella 1 Punteggi conseguiti nelle prove, espressi in percentuale

\begin{tabular}{lrrr}
\hline Classe & \multicolumn{3}{c}{ Abilità } \\
\cline { 2 - 4 } & Ascolto & Audio-video & Testo scritto \\
\hline Terza & 56 & 39 & 80 \\
Quarta & 62 & 41 & 58 \\
Quinta & 43 & 43 & 43 \\
\hline
\end{tabular}


Tabella 2 Statistica descrittiva risultati, classe terza

\begin{tabular}{lccccccrr}
\hline & \multicolumn{2}{c}{ Ascolto } & & & \multicolumn{2}{c}{ Audio-video } & & \multicolumn{2}{c}{ Testo scritto } \\
\cline { 2 - 3 } & Media & DS & & Media & DS & & Media & D S \\
\hline CO-T T & 9,45 & 2,01 & & 6,92 & 2,33 & & 10,30 & 1,73 \\
Classe II & 6,58 & 2,35 & & 5,90 & 2,73 & & 9,62 & 2,18 \\
\hline
\end{tabular}

Tabella 3 Statistica descrittiva risultati, classe quarta

\begin{tabular}{|c|c|c|c|c|c|c|}
\hline & \multicolumn{2}{|c|}{ Ascolto } & \multicolumn{2}{|c|}{ Audio-video } & \multicolumn{2}{|c|}{ Testo scritto } \\
\hline & Media & DS & Media & DS & Media & DS \\
\hline CO-T T & 7,18 & 2,24 & 8,05 & 2,62 & 9,20 & 2,80 \\
\hline Classe IV & 7,46 & 2,07 & 6,00 & 1,93 & 8,17 & 2,17 \\
\hline
\end{tabular}

Tabella 4 Statistica descrittiva risultati, classe quinta

\begin{tabular}{lccccccrr}
\hline & \multicolumn{2}{c}{ Ascolto } & & & \multicolumn{2}{c}{ Audio-video } & & \multicolumn{2}{c}{ Testo scritto } \\
\cline { 2 - 3 } & Media & DS & & Media & DS & & Media & D S \\
\hline CO-T T & 7,84 & 2,52 & & 9,43 & 2,34 & & 9,90 & 2,50 \\
Classe V & 5,17 & 1,90 & & 6,38 & 2,29 & & 6,00 & 2,68 \\
\hline
\end{tabular}

dell'età. Si tratta dell'unica prova in cui la quinta ha raggiunto il risultato migliore fra le tre classi.

Nelle tabelle 2, 3 e 4 saranno presentati i risultati per ciascuna classe. Saranno riportati anche i risultati dei campioni normativi utilizzati per la standardizzazione delle prove, riportati nei manuali del trattamento coT T (Carretti et al. 2013) e delle prove M T (Cornoldi, Colpo e Carretti 2017).

A commento dei dati possiamo osservare che in terza erano presenti tre alunni stranieri al primo anno di scolarizzazione in lingua italiana: data l'esiguità numerica del gruppo classe, la loro ancor scarsa padronanza linguistica potrebbe aver inciso sul risultato complessivo osservato nella prova di ascolto. Per converso, la terza ha invece ottenuto i risultati migliori nella prova di comprensione del testo scritto, con l'8o\% di risposte corrette rispetto alla quarta (58\%) e alla quinta (43\%). Oltre alla diversità di caratteristiche delle prove per la terza, risultate più semplici anche per il campione di standardizzazione, una possibile spiegazione del risultato chiama in causa il ruolo della sperimentatrice, che era anche insegnante della classe e le cui aspettative potrebbero aver agito nelle modalità descritte dalla nota ricerca sul cosiddetto «effetto Pigmalione» (Rosenthal e Jacobson 1991) in base alle quali l'aspettativa di un individuo riguardo al comportamento di un altro può diventarne una predizione semplicemente per il fatto di essere 
stata formulata. Va anche segnalato che, nella prova di comprensione del testo scritto, due alunni stranieri di terza hanno ottenuto punteggi altissimi (12 e 11 punti rispettivamente) il che dimostra le loro abilità nonostante le possibili barriere linguistiche. Si può formulare l'ipotesi che durante l'anno scolastico gli alunni abbiano appreso velocemente la lingua usando diverse strategie implicanti l'uso della propria madre lingua, oppure che la loro motivazione sia elevata in quanto l'italiano è un mezzo comunicativo indispensabile per rapportarsi con gli altri nell'ambiente circostante. Nelle prove d'ascolto e audio-video, invece, la loro prestazione potrebbe essere stata inferiore, poiché queste due abilità richiedono una buona padronanza della lingua per cogliere con immediatezza il significato assunto da una parola all'interno del contesto in cui compare.

Gli alunni della quarta hanno ottenuto risultati migliori nella prova d'ascolto, con il $62 \%$ di risposte esatte. Seguono la prova di comprensione del testo scritto (58\%) e la prova audio-video ( $41 \%)$. Gli alunni della classe potrebbero avere svolto meglio la prova d'ascolto in confronto alle altre due abilità a causa di una maggiore responsività al trattamento sperimentale proposto, o per aver vissuto il testo audiovisivo e quello scritto come scarsamente motivanti.

Come si può notare dalle tabelle, la percentuale delle risposte esatte per la quinta è del $43 \%$ in tutte le prove. Non sono emerse differenze fra le tre abilità, il risultato è inferiore alle aspettative e parzialmente diverso dalle nostre ipotesi, in base alle quali l'abilità di comprensione dovrebbe crescere con l'età. La bassa prestazione della quinta potrebbe essere ricondotta anche alla circostanza che nella classe ospitava alunni con difficoltà di apprendimento scolastico certificate, e altri due alunni in fase di richiesta di certificazione. Le difficoltà oggetto delle certificazioni riguardavano in particolare la competenza comunicativa e le abilità cognitive, di importanza cruciale per la padronanza delle abilità metacognitive di cui il trattamento sperimentale si proponeva la stimolazione. Il risultato ottenuto potrebbe comunque essere dipeso anche dall'esiguità numerica del gruppo di partecipanti, nonché dal carattere non longitudinale della rilevazione.

La tabella 5 presenta i risultati ottenuti dai gruppi sperimentali, suddivisi per classe e genere.

Osservando la media dei punteggi ottenuti dagli alunni, possiamo vedere che nella maggior parte delle prove i maschi hanno riportato punteggi medi più elevati delle femmine. Un motivo per cui i maschi si sono dimostrati più abili nello svolgimento delle prove potrebbe essere ricondotto al fatto che gli alunni sembrano riuscire a gestire l'ansia meglio delle alunne. 
Tabella 5 Punteggi delle prove per singole classi e genere

\begin{tabular}{|c|c|c|c|c|c|c|}
\hline & \multicolumn{2}{|c|}{ Ascolto } & \multicolumn{2}{|c|}{ Audio-video } & \multicolumn{2}{|c|}{ Testo scritto } \\
\hline & $\mathrm{M}$ & $\mathrm{F}$ & $\mathrm{M}$ & $\mathrm{F}$ & $\mathrm{M}$ & F \\
\hline Terza & 6,50 & 6,60 & 8,00 & 5,38 & 10,00 & 9,50 \\
\hline Quarta & 7,50 & 7,33 & 5,00 & 6,86 & 9,40 & 7,29 \\
\hline Quinta & 5,44 & 4,33 & 6,33 & 6,50 & 6,67 & 4,50 \\
\hline
\end{tabular}

Essi dimostrano di avere padronanza di sé in situazioni scolastiche impegnative, fronteggiano con coraggio le prove e rispondono meglio allo stress che nasce dal confronto o dalla presenza di vincoli di tempo per la risoluzione di un test. Le alunne, invece, tendono a divenire ansiose più facilmente, e affrontano con minore lucidità circostanze difficili o complesse. Un ulteriore fattore che potrebbe spiegare il risultato ottenuto è il fatto che i maschi tendono a mettere in relazione la vita quotidiana con ciò che studiano in classe, e mostrano maggiore interesse verso approcci riconducibili a competenze utilizzabili nella pratica (Zanniello 2013). Oltre a ciò, i temi delle prove potrebbero essere risultati più consoni ai bambini che alle bambine.

\section{Esempi di analisi dei distrattori}

Oltre all'analisi generale fin qui discussa, si è stabilito di eseguire un'analisi delle risposte formulate dai partecipanti a ciascuno degli item di cui si componevano le prove a scelta multipla somministrate. L'analisi ha inteso verificare la presenza di item complessi, ambigui o in grado di far emergere lacune legate alla particolare situazione di competenza linguistica dei partecipanti; queste ultime si sono manifestate, in alcuni casi, nella prevalente scelta di distrattori (risposte errate) in luogo delle alternative corrette (Falocci et al. 2010).

Nei limiti del tutto esplorativi del lavoro qui presentato, si ritiene di poter ricondurre le lacune evidenziate dalle risposte errate dei partecipanti a tre situazioni di criticità, su cui intervenire nella programmazione didattica:

1. criticità legate a difficoltà di comprensione del lessico;

2. criticità legate a difficoltà di comprensione del significato dei connettivi;

3. criticità legate a difficoltà di costruzione della coerenza testuale.

Saranno ora presentati e discussi alcuni item in cui, per ciascuna classe, 
l'azione dei distrattori è stata più evidente. In ciascun esempio, la risposta corretta è evidenziata con un asterisco, e accanto a ciascuna alternativa di risposta è indicato tra parentesi il numero di alunni da cui è stata scelta.

\section{Esempi di criticità legate a difficoltà di comprensione del lessico}

Esempio 1: classe IV, ascolto, item 3 - lessico (microlingua)

Il «tefra» è:
A il magma eruttato dal vulcano (5)
B il materiale eruttato in cielo (3)*
$C$ il fumo che esce dal vulcano (1)
D la lava quando si solidifica (4)

Il risultato potrebbe essere dovuto all'influenza esercitata da conoscenze di sfondo, che permettono di collegare il testo con le proprie esperienze di vita dandovi coerenza. Il termine tecnico tefra, di impiego non frequente, non è stato associato alle ceneri, ma ad altri prodotti vulcanici identificati con termini maggiormente noti e più agevolmente disponibili.

\section{Esempio 2: classe V, ascolto, item 1 - lessico (derivazione con suffissi)}

La parola «gladio» significa:
A gladiatore che combatte con una spada corta (5)
B una spada corta usata nel combattimento a distanza (2)
C una spada corta usata nel combattimento ravvicinato (2)*
D una spada lunga utilizzata nel corpo a corpo (3)

Gli alunni potrebbero non avere risposto correttamente in quanto hanno associato la parola da definire (gladio) con la parola "gladiatore», che appartiene alla stessa famiglia di parole, ma ha un diverso significato. Emergerebbe allora una difficoltà degli alunni nell'utilizzo di regole di derivazione lessicale implicanti l'uso di alcuni suffissi.

\section{Esempio 3: classe V, ascolto, item 9 - lessico (significato avverbi)}

Quale fu la conseguenza della tradizione cristiana sulle lotte dei gladiatori?
A furono comunque sempre tollerate (3)
B furono proibite ai fedeli (4)
C furono eliminate immediatamente per tutti (4)
D furono progressivamente eliminate (1)* 
All'item ha risposto correttamente un solo alunno, mentre gli altri potrebbero aver incontrato delle difficoltà nella comprensione del significato dell'avverbio progressivamente, scegliendo di conseguenza gli altri descrittori. Si nota una mancata competenza linguistica nel distinguere il significato dei due avverbi (progressivamente/immediatamente), in ragione della quale la sinonimia tra i due verbi eliminare e proibire potrebbe avere indotto alla scelta del distrattore B, compatibile con l'atteggiamento negativo dei cristiani verso i combattimenti.

\section{Esempi di criticità legate a difficoltà di comprensione del significato dei connettivi \\ Esempio 4: classe IV, ascolto, item 9 - comprensione connettivi, connettivo concessivo}

Il vulcano Kilauea delle isole Hawai è un vulcano:

A sicuro perché ormai inattivo (6)

B sicuro anche se ancora attivo (o)*

C interessante per i turisti, ma pericoloso (3)

D molto frequentato, ma difficile da raggiungere (3)

Le risposte date dagli alunni potrebbero documentare una difficoltà nel comprendere l'uso della congiunzione concessiva (anche se) rispetto alla congiunzione causale (perché): nessuno degli alunni, infatti, ha scelto l'alternativa di risposta corretta (B). Non comprendendo il significato del connettivo concessivo, gli alunni potrebbero allora aver interpretato l'informazione in riferimento a un nesso di tipo causale.

\section{Esempio 5: classe V, ascolto, item n. 11 - comprensione connettivi, connettivo avversativo-concessivo}

Le condizioni di vita dei gladiatori erano:
A molto buone (o)*
B quelle dei comuni schiavi (5)
C simili a quelle dei soldati (3)
D simili a quelle dei prigionieri (4)

Le risposte date dagli alunni potrebbero documentare una difficoltà nel comprendere il significato della congiunzione avversativa-concessiva tuttavia. La frase ascoltata si presenta nel seguente modo: «La carriera di un 
gladiatore era pericolosa e spesso di breve durata. Tuttavia i campioni godevano di privilegi che uno schiavo normale non poteva nemmeno immaginare» (Carretti et al. 2013). Non comprendendo il valore della congiunzione "tuttavia», gli alunni potrebbero aver associato le condizioni di vita dei gladiatori a quelle di schiavi, soldati e prigionieri, categorie proposte nelle risposte diverse da quella corretta.

\section{Esempi di criticità legate a difficoltà di costruzione della coerenza testuale}

\section{Esempio 6: classe IV, testo scritto, item 10}

Il panda mangia molto bambù perché:
A ha molta fame (o)
B non c'è altro da mangiare (2)
$\mathrm{C}$ il bambù è un alimento poco nutriente $(5)^{*}$
D deve accumulare grasso per l'inverno (5)

Metà degli alunni ha scelto la risposta $\mathrm{D}$, che rinvia a una conoscenza generale (per sopravvivere all'inverno bisogna accumulare riserve, quindi mangiare molto cibo) non specificamente legata alle informazioni contenute nel testo, che riguardano il regime alimentare vegetariano del panda.

\section{Esempio 7: classe IV, testo scritto, item 11}

Il panda vive soprattutto in Cina perché:
A lì è vicino allo zoo di Pechino (o)
B lì trova il suo cibo preferito (4)*
$C$ in Cina ci sono molti alberi dove si può arrampicare (3)
D è il simbolo scelto dal WWF (4)

La domanda intende verificare la capacità degli alunni di utilizzare conoscenze precedenti o contestuali per trarre inferenze semantiche nel testo. Il risultato sembrerebbe indicare una lettura poco attenta del testo da parte degli alunni, e il conseguente prevalere di strategie di comprensione basate sul recupero di informazioni a esso estranee e irrilevanti.

\section{Esempio 8: classe V, ascolto, item 10}

Il successo di pubblico delle lotte fra gladiatori nell'antica Roma mostra che i Romani amavano: 

A la ricchezza (6)
B la forza fisica (5)*
C gli agi (1)
D la cultura (o)

Dalle risposte scelte emerge che gli alunni hanno collegato il termine «Romani» alla loro condizione di ricchezza e agiatezza, menzionate nel testo, ma irrilevanti ai fini della risposta. Ciò ci induce a ipotizzare che gli alunni non abbiano letto attentamente il quesito o prestato attenzione durante l'ascolto, e abbiano utilizzato le proprie conoscenze pregresse per rendere coerente il significato del testo.

\section{Discussione e conclusione}

L'obiettivo del presente contributo era di valutare le abilità di comprensione dell'ascolto e del testo scritto, nonché la capacità degli alunni di cogliere il contenuto di informazioni presenti in un testo, o da esso inferibili. L'esigenza di valutare queste abilità è motivata dal fatto che le abilità ricettive sono un aspetto fondamentale nel processo di apprendimento, e costituiscono una condizione necessaria per il successo nell'apprendimento in tutte le discipline scolastiche.

I dati raccolti mostrano che i punteggi ottenuti nelle tre classi, in tutte le abilità, sono più bassi rispetto a quelli del campione di standardizzazione delle prove somministrate. Tale risultato era prevedibile e costituiva una delle ipotesi della nostra ricerca: gli alunni che frequentano le scuole con lingua d'insegnamento italiana in Slovenia, infatti, vivono in un contesto diverso da quello italiano, nel quale sono state determinate le fasce di prestazione standardizzate.

Le prove che hanno valutato la comprensione dell'ascolto hanno ottenuto risultati inferiori rispetto alla prova di comprensione del testo scritto, il che ci induce a sottolineare l'importanza del potenziamento di questa abilità già nei primi anni della scolarizzazione. La priorità delle abilità ricettive su quelle produttive, e la priorità delle abilità di ricezione orale su quella scritta, sono del resto condivise anche dalla riflessione glottodidattica e stanno alla base dell'individuazione degli obiettivi dell'educazione linguistica.

I dati ottenuti non permettono di convalidare l'ipotesi riguardante il miglioramento della comprensione in tutte le prove al crescere dell'età. Nella prova di comprensione dell'ascolto i risultati migliori sono stati ottenuti in quarta, nella prova audio-video in quinta e nella prova di comprensione del testo scritto in terza. Ciò potrebbe essere dovuto al fatto che nella quinta 106 
erano inclusi cinque alunni con bisogni educativi speciali (B E S), di cui tre certificati e due in fase di certificazione; i punteggi di questi alunni, data anche l'esiguità numerica delle classi, potrebbero aver influito sul risultato medio generale.

Gli alunni di terza hanno registrato risultati lodevoli, soprattutto nella prova di comprensione del testo scritto: ciò potrebbe essere dovuto al fatto che l'insegnante era direttamente coinvolta nella ricerca in quanto sperimentatrice. In questo senso, il risultato conferma ulteriormente l'importanza che i docenti adottino un atteggiamento incoraggiante e positivo verso i progressi di tutti i propri alunni, sulla scorta del seminale e già citato lavoro di Rosenthal e Jacobson (1991).

Nella ricerca abbiamo analizzato anche l'eventuale presenza di differenze legate al genere, rilevando una migliore prestazione dei maschi rispetto alle femmine. Il risultato ottenuto è meritevole di ulteriore approfondimento, poiché non coincide con quanto attestato dalla ricerca sulla comprensione della lettura. Un'ipotesi per spiegare il risultato ottenuto è che i testi proposti siano risultati impegnativi e motivanti per i maschi, che avrebbero mobilitato le proprie risorse attentive e cognitive, riuscendo anche ad affrontare con minore preoccupazione una prova caratterizzata da un tempo di esecuzione limitato.

L'analisi delle risposte ai singoli item ha permesso di formulare ipotesi sugli aspetti linguistici e metacognitivi da promuovere e valorizzare, da parte dei docenti, nei processi di insegnamento e apprendimento. Dai risultati emerge che gli alunni hanno maggiori difficoltà nella comprensione e manipolazione di taluni aspetti del lessico, di qualche avverbio e di alcuni connettivi, in particolare di tipo concessivo. Un altro aspetto emerso nell'esame a posteriori delle risposte alle prove è la mancanza di efficaci strategie di ristabilimento della coerenza testuale locale: in queste situazioni, gli alunni tendono a trascurare informazioni presenti nei testi, e rilevanti per la comprensione, e ricorrono a integrazioni basate su conoscenze enciclopediche, o su elementi testuali non rilevanti.

Dal punto di vista metodologico, la presente ricerca presenta alcuni limiti non trascurabili. Nel piano della ricerca non è stata prevista la somministrazione di un pre-test riguardante le prestazioni degli alunni nelle abilità oggetto di rilevazione; inoltre, l'esiguità numerica della popolazione da cui sono stati individuati i partecipanti non ha consentito la costituzione di gruppi di controllo. Per tali ragioni, i risultati e le osservazioni formulate riguardano un ristretto insieme di dati, non possono costituire in alcun modo la base per generalizzazioni dotate di validità statistica e vanno intese solo come presupposto esplorativo per la formulazione di ul- 
teriori ipotesi di lavoro. Ciò detto, vorremmo comunque ribadire che l'analisi e l'interpretazione dei dati riguardanti le competenze negli ambiti della comprensione dell'ascolto e del testo scritto ci pare utile, in un contesto caratterizzato da marcato plurilinguismo e da differenti livelli di competenza nella lingua italiana, per indicare ai docenti - con il supporto di evidenze empiriche - alcune direzioni di intervento.

I risultati del lavoro supportano la proposta, rivolta ai docenti in servizio, di adottare pratiche di insegnamento e strumenti di analisi simili a quelli qui presentati, per i benefici che essi possono portare sia al miglioramento dell'offerta didattica sia al potenziamento delle competenze linguistiche degli alunni.

\section{Riferimenti bibliografici}

Albanese, O., P. Doudin e D. Martin. 2003. Metacognizione ed educazione: processi, apprendimenti, strumenti. Milano: Franco Angeli.

Balboni, P. 1994. Didattica dell'italiano a stranieri. Roma: Bonacci.

- 2007. Tecniche didattiche per l'educazione linguistica: italiano, lingue straniere, lingue classiche. Novara: De Agostini Scuola.

- 2012. Le sfide di Babele: insegnare le lingue nelle società complesse. Novara: De Agostini Scuola.

Boscolo, P. 2006. Psicologia dell'apprendimento scolastico: aspetti cognitivi e motivazionali. Torino: U TE T.

Carretti, B., C. Cornoldi, N. Caldarola e C. Tencati. 2013. CO-T T: comprensione orale; test e trattamento. Trento: Erickson.

Cornoldi, C., e C. Caponi. 1991. Memoria e metacognizione: attività didattiche per imparare a ricordare. Trento: Erickson.

Cornoldi, C., G. Colpo e B. Carretti. 2017. Prove M T: kit scuola primaria; dalla valutazione degli apprendimenti di lettura e comprensione al potenziamento. Firenze: Giunti.

Crasnich, S. 2019. «Acquisizione linguistica precoce e ruolo dell'adulto: la formazione in servizio come occasione di crescita professionale.» In $\mathrm{Vz}$ goja in izobraževanje predšolskih otrok prvega starostnega obdobja, a cura di S. Čotar Konrad, B. Borota, S. Rutar, K. Drlijć e G. Jelovčan, 263-276. Capodistria: Založba Univerze na Primorskem.

Daloiso, M. 2009. I fondamenti neuropsicologici dell'educazione linguistica. Venezia: Cafoscarina.

- 2011. Introduzione alla didattica delle lingue moderne: una prospettiva interdisciplinare. Roma: Aracne.

Falocci, N., M. Gnaldi, M. Matteucci e S. Mignani. 2010. «La validazione statistica dei test standardizzati di profitto: principali aspetti di metodo e due casi di studio sulla valutazione degli apprendimenti nella scuola 
primaria.» Working Paper 09/2010, Istituto Nazionale per la Valutazione del Sistema Educativo di Istruzione e di Formazione. http://amsacta .unibo.it/2875/1/wpo9_Falocci.pdf.

Ferraboschi, L., e N. Meini. 1993. Strategie semplici di lettura: esercizi guida per la comprensione del testo. Trento: Erickson.

Ianes, D. 1996. Metacognizione e insegnamento: spunti teorici e applicativi. Trento: Erickson.

Mikolič, V., S. Pertot e N. Zudič Antonič. 2006. Med kulturami in jeziki/Tra lingue e culture. Capodistria: Annales.

Rosenthal, R., e L. Jacobson. 1991. Pigmalione in classe: aspettative degli insegnanti e sviluppo intellettuale degli allievi. Milano: Franco Angeli.

Zanetti, M. A., e D. Miazza. 2004. La comprensione del testo: modelli e ricerche in psicologia. Roma: Carocci.

Zanniello, G. 2013. «Didattica e differenze di genere in alunni di 8-12 anni.» ECPS Journal, n. 7, 85-114.

Zudič Antonič, N. 2007. «L'educazione letteraria in prospettiva interculturale.» Tesi di dottorato, Università Ca' Foscari.

\section{Preverjanje slušnega in bralnega razumevanja v osnovni šoli}

Opisani prispevek predstavlja kvantitativno raziskavo, ki temelji na preverjanju slušnega in bralnega razumevanja pri učencih, ki obiskujejo osnovno šolo $\mathrm{z}$ italijanskim učnim jezikom v Slovenski Istri. Tema izhaja iz pomena slušne in bralne zmožnosti v procesu poučevanja in učenja. Empirični del prispevka vključuje analizo rezultatov testov, ki smo jih uporabili v posameznem razredu in njihovo interpretacijo. $V$ zaključnem delu je predstavljena analiza posameznih odgovorov, ki so jih učenci podali pri reševanju testa.

Ključne besede: poučevanje jezika, receptivne dejavnosti, razumevanje, standardizirani testi, analiza

\section{Assessment of Listening and Reading Comprehension in the Elementary School}

The paper presents a quantitative research on listening and reading comprehension of primary school pupils attending a school with Italian as language of instruction on the Slovene Coast. This topic was chosen due to the importance of listening and reading comprehension skills in the process of teaching and learning. The empirical part of the paper includes the analysis of tests administered in each class and the interpretation of results. The paper concludes with the analysis and the discussion of selected answers given by pupils.

Key words: language education, receptive abilites, comprehension, standardized tests, analysis 